\title{
A Systematic Review of the Effect of Expectancy on Treatment Responses to Acupuncture
}

\author{
Ben Colagiuri' ${ }^{1,2}$ and Caroline A. Smith ${ }^{1}$ \\ ${ }^{1}$ Centre for Complementary Medicine Research, University of Western Sydney, NSW 2751, Australia \\ ${ }^{2}$ School of Psychology, University of New South Wales, Kensington, NSW 2052, Australia
}

Correspondence should be addressed to Ben Colagiuri, b.colagiuri@unsw.edu.au

Received 25 May 2011; Revised 9 August 2011; Accepted 6 September 2011

Academic Editor: David Baxter

Copyright (C) 2012 B. Colagiuri and C. A. Smith. This is an open access article distributed under the Creative Commons Attribution License, which permits unrestricted use, distribution, and reproduction in any medium, provided the original work is properly cited.

\begin{abstract}
Randomised controlled trials (RCTs) of acupuncture often find equivalent responses to real and placebo acupuncture despite both appearing superior to no treatment. This raises questions regarding the mechanisms of acupuncture, especially the contribution of patient expectancies. We systematically reviewed previous research assessing the relationship between expectancy and treatment responses following acupuncture, whether real or placebo. To be included, studies needed to assess and/or manipulate expectancies about acupuncture and relate these to at least one health-relevant outcome. Nine such independent studies were identified through systematic searches of Medline, PsycInfo, PubMed, and Cochrane Clinical Trials Register. The methodology and reporting of these studies were quite heterogeneous, meaning that meta-analysis was not possible. A descriptive review revealed that five studies found statistically significant effects of expectancy on a least one outcome, with three also finding evidence suggestive of an interaction between expectancy and type of acupuncture (real or placebo). While there were some trends in significant effects in terms of study characteristics, their generality is limited by the heterogeneity of study designs. The differences in design across studies highlight some important methodological considerations for future research in this area, particularly regarding whether to assess or manipulate expectancies and how best to assess expectancies.
\end{abstract}

\section{Introduction}

Many studies comparing real acupuncture to placebo controls fail to find statistically significant differences between these two treatments but often find that both real acupuncture and the placebo controls produce better outcomes than no treatment or standard care alone [1-4]. This suggests that there is some benefit to providing acupuncture treatment, whether real or placebo, but raises questions about the underlying mechanisms of these effects. The three most common explanations proposed to account for improvements following both real and placebo acupuncture are that (1) needling is only one of a variety of active components in acupuncture treatment, (2) the placebo controls used in the studies are, in fact, active treatments and, therefore, invalid placebos, or (3) improvement following both real and placebo acupuncture results from the placebo effect.
Placebo (or sham) control in randomised placebo-controlled trials (RCTs) involves comparing the therapy of interest with a dummy treatment so that all participants engage in a treatment process, but only those allocated to the target therapy receive the specific component being tested [5]. Acupuncture is a complex intervention involving diagnosis, needling, facilitating patients active involvement in their recovery, lifestyle advice, and therapeutic alliance, all of which are tailored individually to the patient being treated [6]. Some researchers have argued that these components cannot be validly partitioned and that assessing individual components will underestimate the true efficacy of acupuncture, because the response to the whole acupuncture intervention may be greater than the sum of responses to the components of acupuncture administered individually [610]. If so, this means that RCTs, which seek to isolate and test the efficacy of a single component, may not be appropriate 
for assessing acupuncture. This would suggest that a lack of difference between real and placebo acupuncture in RCTs may result from the omission of important components of acupuncture, such as facilitating patients active involvement in their recovery and lifestyle advice, that is common in these trials $[6,11]$. However, before such a conclusion can be drawn, evidence is required that demonstrates a larger benefit of providing acupuncture treatment than summing the benefit of providing the individual components of acupuncture alone, which, to our knowledge, has not yet been tested.

Placebo (or sham) controls adopted in RCTs of acupuncture include needle insertion at nonacupuncture points (sham acupuncture), shallow needle insertion that does not penetrate below the skin (minimal or superficial needling), and blunt needles that touch, but do not penetrate the skin (placebo needling). Lundeberg and colleagues [12-14] have argued that these techniques are not inert and are, therefore, invalid as placebo controls. They provide a list of eleven reasons why the placebo controls used in acupuncture RCTs may be active treatments, including evidence of physiological responses to sham acupuncture, evidence that superficial and sham needling producing larger effects than a placebo pill, and, rather strangely, that placebo controls can be as effective or even more effective than real acupuncture.

However, the evidence provided by Lundeberg et al. [14] can be explained equally well in the context of patient expectancies. Expectancy is proposed to be a key mechanism of the placebo effect. Placebo effects are changes that occur in response to receiving treatment but that are not due to the inherent properties of the treatment itself [15]. Many studies have found that a saline injection or placebo cream administered under the guise of a powerful analgesic can, in fact, reduce pain, for example [16-22]. There is also evidence for placebo effects across a range of other conditions (see [23] for a recent review). For example, placebo treatment appears to reduce depressive symptoms [24], improve sleep quality [25] improve motor performance in patients with Parkinson's disease [17], modulate heat rate in healthy volunteers [17], and improve cognitive performance in healthy volunteers [26]. Perhaps most interestingly, Benedetti et al. [27] found significantly larger treatment effects for postoperative pain, motor performance in patients with Parkinson's disease, and heart rate in healthy participants when the initiation of treatment was signalled to the patient by a health professional compared with when it was initiated surreptitiously without the patients' awareness, indicating that most medical treatments involve a placebo component. On this basis, some researchers have argued that the superiority of both real and placebo acupuncture techniques over no treatment (or in some cases standard care) combined with failure to find significant differences between real and placebo acupuncture can be explained by the placebo effect $[28,29]$. That is, they argue that any improvement following acupuncture treatment, whether real or placebo, results from the patients expecting acupuncture to be effective.

If expectancies do lead to real changes in symptoms via the placebo effect, then physiological changes must underlie these effects. Therefore, the physiological changes Lundeberg et al. [14] cite following placebo acupuncture do not discount the possibility of expectancy effects. There is also evidence that the more invasive the placebo, the larger the placebo effect. For example, four placebo pills reduced recovery times from duodenal ulcers compared with two placebo pills [30] and a subcutaneous placebo injection reduced pain due to migraine headaches more effectively than a placebo pill [31]. As such, placebo acupuncture may simply produce stronger expectancy effects than placebo pills do. Finally, if both real and placebo acupuncture exert their effects as a result of expectancy, then this would lead to frequent null differences and occasional statistically significant differences between the two treatments caused by sampling variation (cf. Type I error [32]), including placebo acupuncture appearing superior to real acupuncture on occasion As a result, there is as yet no conclusive evidence that the currently used placebo controls are active beyond expectancy.

Perhaps more importantly, the three alternative explanations for the common lack of statistically significant differences between real and placebo acupuncture are not mutually exclusive. Needling may be more efficacious when delivered with lifestyle advice, but this does not mean that patients' expectancies about the efficacy of an acupuncture intervention cannot influence their outcomes via the placebo effect. Similarly, currently used placebo controls for acupuncture needling could be invalid, but this does not preclude the possibility that expectancies could contribute to responses to real acupuncture. As demonstrated by Benedetti et al. [27], most medical treatments, whether efficacious or not, appear to be influenced by patient expectancies. Thus, regardless of whether or not the combined effects of an acupuncture intervention cannot be explained by the effects of each component's individual efficacy or whether or not the currently used placebo controls in acupuncture RCTs are valid, it remains important to establish both if and how the placebo effect contributes to responses to acupuncture.

With this in mind, we conducted a systematic review of the literature to examine whether expectancies can influence acupuncture outcomes. Although we had intended to use meta-analysis to estimate and test the magnitude of the effect of expectancy on treatment responses following acupuncture, the studies identified were too heterogeneous with respect to methodology and reporting to allow such analysis. We, therefore, provide a descriptive review of studies investigating placebo effects in acupuncture, drawing particular attention to methodological considerations, and outline some key goals for future research in this area.

\section{Methods}

2.1. Search Strategy. Articles were identified through computerized literature searches. Medline, PsycInfo, PubMed, and Cochrane Clinical Trials Register were searched for English publications from inception up to 1st December, 2010 using the search terms "expectancy OR expectancies OR expectation\$ OR expected efficacy OR placebo effect\$” in combination with "acupuncture" using title and abstract fields. The reference lists of publications identified through 
the electronic search were also screened for additional relevant articles.

2.2. Selection Criteria. To be included, studies needed to either assess or manipulate participants' expectancies regarding the efficacy of an acupuncture intervention involving needling and to report on the relationship between these expectancies or the manipulation and at least one outcome variable. The acupuncture intervention could include manual or electroacupuncture and could be standardised or individualised. Assessing expectancies regarding the efficacy of acupuncture involved any question asking participants to rate their expectancies for improvement as a result of acupuncture but had to be prospective; that is, the expectancy assessment had to occur before the acupuncture treatment. Manipulating expectancies meant allocating participants to receive different information about the likely effects of their treatment, whether real or placebo acupuncture was delivered. For example, Suarez-Almazor et al. [33] randomly allocated participants in a RCT comparing real and sham acupuncture for osteoarthritis of the knee to receive suggestion from the acupuncturist that either the treatment "will work" (high expectancy) or that it "may or may not work" (low expectancy). Studies investigating both clinical and nonclinical conditions (e.g., experimentally-induced pain) were included. The studies could assess any healthrelated outcome, whether subjective or objective, and there were no constraints on study design, as long as the criteria for assessing and/or manipulating expectancies were met. Only peer-reviewed publications in English were included.

2.3. Study Selection. One author (B. Colagiuri) conducted the initial search and excluded articles that were clearly not relevant. Both authors then reviewed the full texts of each of the remaining articles and evaluated them against the selection criteria independently. Any disagreements were resolved through discussion.

The literature search identified a total of nine independent studies reporting on the relationship between expectancy and treatment response following acupuncture suitable for inclusion. Figure 1 displays the flow diagram for study selection. The search of Medline, PsycInfo, PubMed, and Cochrane Clinical Trials Register provided a total of 392 English references. After removing duplicates, there were 201 articles, of which 184 were clearly not relevant. The full texts of the remaining 17 articles were reviewed independently by both authors. Of these, three articles were excluded because their results were reported in other articles already identified [34-36]. This left 14 unique studies. One article was excluded because it reported on the relationship between expectancy and acupuncture combined with expectancy and an exercise intervention [37]. One article was excluded because no details of the expectancy assessment were provided [38]. One was excluded because it focused on patients with psychological comorbidity [39], which although not an a priori exclusion criteria, both authors agreed might affect the relationship between expectancy and treatment outcomes. One was excluded because it only assessed participants'

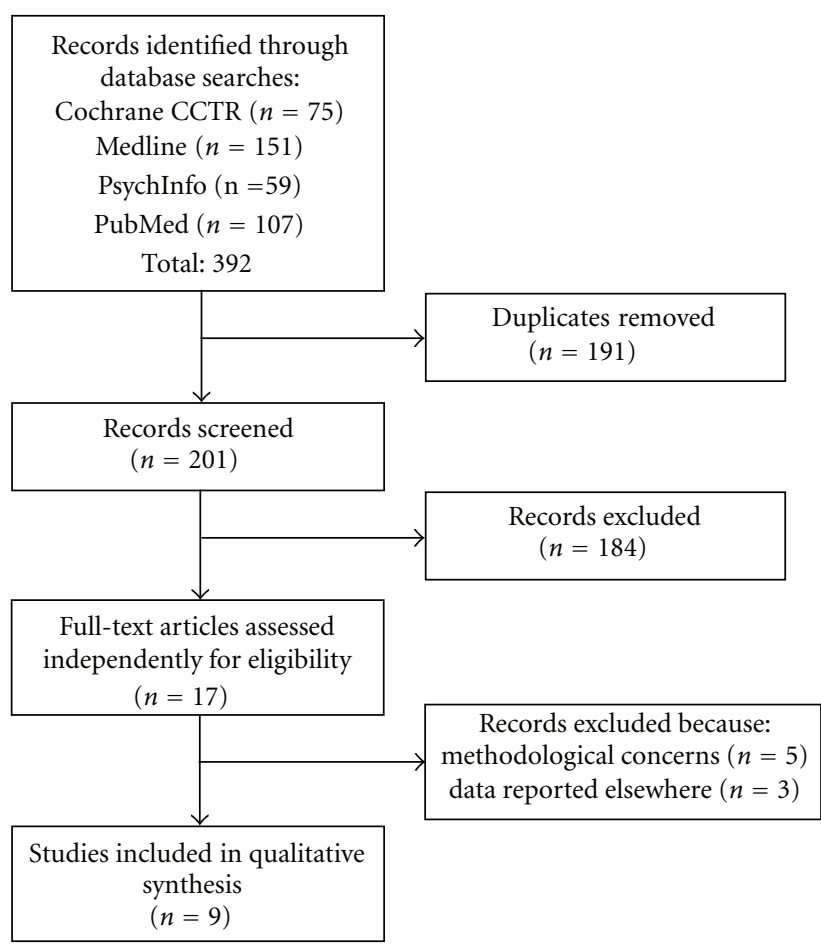

FIgURE 1: Flow diagram for study identification and selection.

expectancies retrospectively in the form of guesses about treatment allocation [29]. One was excluded because it failed to directly test the effect of its expectancy manipulation [40].

2.4. Data Extraction. The authors reviewed the retrieved articles and independently extracted information on sample characteristics, study design, outcome variables, relevant results, and whether the study fulfilled the inclusion criteria using pre-defined coding sheets. The sample characteristics included sample size, proportion of female participants, and whether the participants had previously used acupuncture. Study design included the experimental design, characteristics of the acupuncture treatment that was delivered, and how expectancies were either assessed or manipulated. Study outcomes involved all outcomes that were analysed for relationships with expectancy and were classified into either self-report or objective outcomes. Differences were discussed, and a final assessment was negotiated for each study. The PRISMA guidelines for reporting of systematic reviews and meta-analyses were followed $[41,42]$.

2.5. Risk of Bias Assessment. Scoring studies numerically based on their quality is controversial. This is because combining quality items into a single score is questionable, particularly in terms of whether or not these items are additive $[43,44]$, and because there is evidence that currently used quality scores do not actually predict variance in effect sizes $[45,46]$. We, therefore, chose not to attribute quality scores to the included studies. Instead, we conducted a risk of bias assessment using the Cochrane Collaborations tool for assessing risk of bias [47], which includes six dimensions, 
namely, adequate sequence generation, allocation concealment, blinding, incomplete data, selective reporting, and other forms of bias. Both authors completed the risk of bias assessment for each study independently, with any discrepancies resolved through discussion.

2.6. Data Analysis. Meta-analysis of the studies was not possible due a combination of heterogeneous methodology used across studies and incomplete reporting of results in some studies. Study results were considered statistically significant if $P<0.05$.

\section{Results}

3.1. Study Characteristics. A summary of the characteristics of the nine studies we identified is provided in Table 1. The majority of studies were on pain-related conditions, both clinical [33, 48-51] and experimentally-induced [52-54]. One study focused on angina pectoris [55]. In six of the studies, participants were acupuncture naive [33, 48, 51$54]$, in two studies, participants had not previously received acupuncture for the condition being treated [50, 55], and in one study no information was provided on participants' previous use of acupuncture [49]. Electro acupuncture was used in five studies [33, 52-55], manual acupuncture was used in three studies [48, 49, 51], and one study only investigated placebo acupuncture [50]. Five of the studies assessed expectancies [49-51, 53, 55], four manipulated expectancies [33, 48, 52, 54]. Assessing expectancies generally involved asking participants to rate how effective they expected acupuncture to be for improving their condition on Likert-type scales. In the majority of studies assessing expectancies, participants were either dichotomised into high and low expectancies [49, 53, 55] or trichotomised into high, medium, or low expectancies [51]. Manipulating expectancies typically involved randomising participants to receive information aimed at enhancing their expectancies for improvement following acupuncture or either neutral or negative information although one study used a conditioning procedure [54]. All studies included self-reported outcomes, but three also included objective outcome variables [33, 48, 55].

\subsection{The Effect of Expectancy on Responses to Acupuncture.} Table 2 provides a descriptive summary of each of the nine studies' findings. The results of the studies were clearly mixed, with some studies finding at least some evidence of a statistically significant effect of expectancy on acupuncture outcomes [33, 49, 52-54] and others failing to find any such effects $[48,50,51,55]$. Interestingly, there were also some findings that were suggestive of an interaction between expectancy and type of acupuncture (real versus placebo). For example, Linde et al. [49] found that the improvement in patients classified as having "high expectancy" compared with those classified as having "low expectancy" was significantly more marked in patients receiving real acupuncture compared with placebo acupuncture. However, evidence of this type of interaction was inconsistent across the studies with some studies finding evidence suggestive of an interaction $[49,52,53]$ and others failing to find such evidence $[33,54]$. Interaction effects were either not reported $[48,51,55]$ or not relevant (because only one acupuncture treatment was administered [50]) in the remaining studies. No study found evidence of significant effects of expectancy on objective outcomes following acupuncture; however, only three studies included objective outcome variables [33, 48, 55].

There were some patterns in terms of the study characteristics and whether or not a significant relationship between expectancy and acupuncture outcomes was found. All three studies investigating experimentally-induced pain found evidence of a significant relationship [52-54], whereas only two of the six studies investigating clinical outcomes found evidence of a significant relationship [49, 51]. Three of the four studies that manipulated expectancies found evidence of a significant relationship [33, 52, 54], whereas only one of the five studies that assessed expectancies found evidence of a significant relationship [49]. Four of the five studies involving electroacupuncture found evidence of a significant relationship between expectancies and treatment response [33, 52-54], whereas only one out of the four studies involving manual acupuncture found evidence of such a relationship [49]. A high degree of caution is, however, necessary when attempting to generalise from these patterns as simple vote counting, that is, summing and comparing the number of significant results with the number of nonsignificant results, is associated with a number of problems [56]. In the current case, for example, even though only two of the six studies investigating clinical outcomes found evidence of a significant relationship between expectancy and acupuncture outcomes [33, 49], these were the two largest in terms of sample size and likely had the most statistical power. The same applies to the only study finding a significant relationship that assessed expectancies [49]. It is also worth noting that studies with healthy volunteers in experimental settings should require fewer participants to achieve the same power as studies in clinical settings, because the former are often better able control for potential confounding variables due to the controlled laboratory setting, which further complicates comparison across these studies. Therefore, while it seems clear that expectancies can affect acupuncture outcomes under at least some circumstances, it is difficult to identify which circumstances these are and how strong this relationship is from the available evidence.

3.3. Risk of Bias. As shown in Table 3, all but one study [33] had either some risk or an unclear risk of bias on at least one of the six dimensions assessed. Specifically, sequence generation was inadequate in one study [52] and unclear in four studies [48, 53-55]. Allocation concealment was not used in one study [52] and was unclear in three studies $[48,54,55]$. Participants were blinded to whether or not they were receiving real or placebo acupuncture in all studies, but in four studies the blinding of outcome assessors was unclear $[48,49,53,54]$. All studies satisfactorily addressed incomplete data, and only one had unclear risk regarding selective reporting [55]. In terms of other biases, four studies 


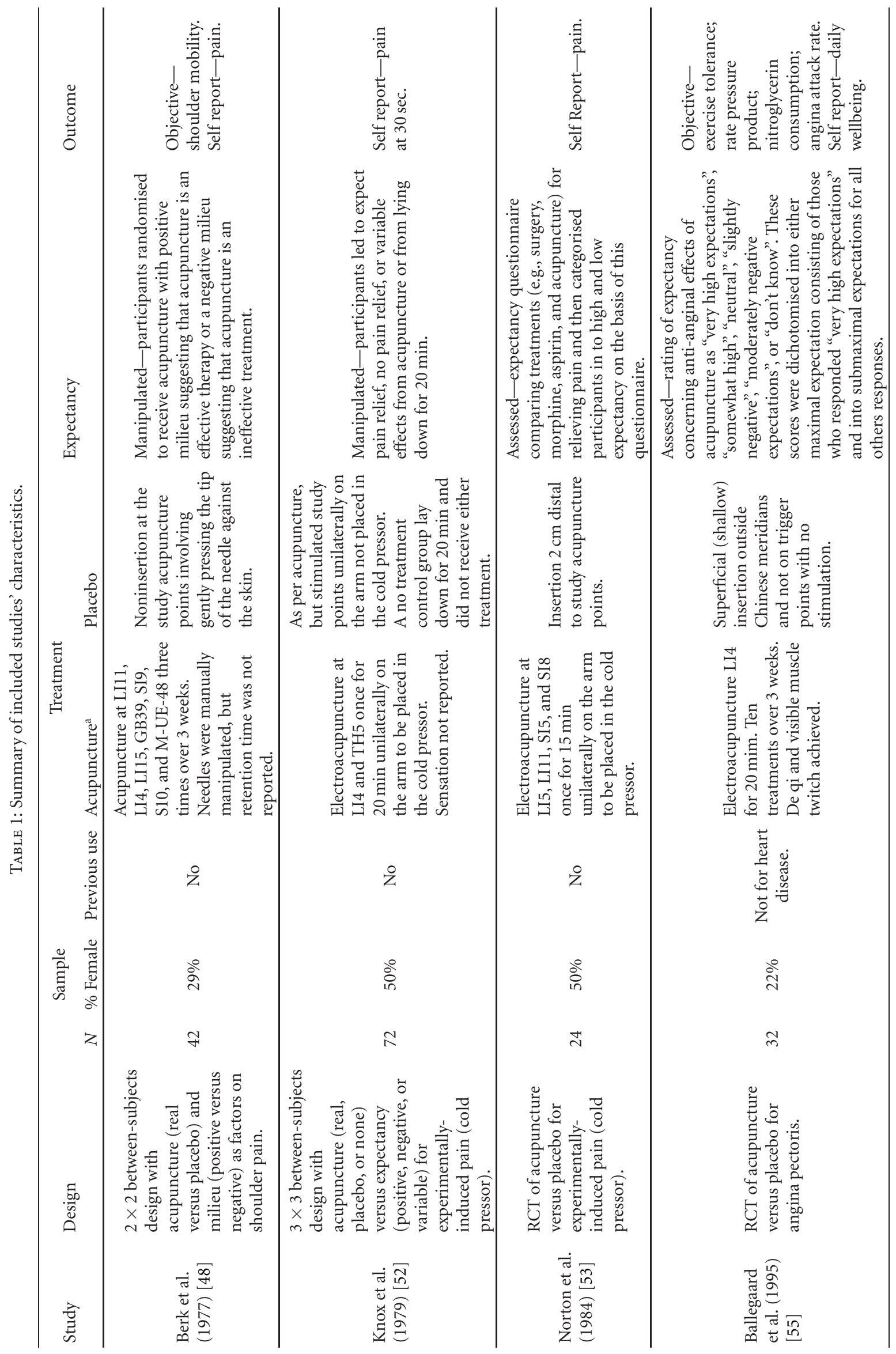




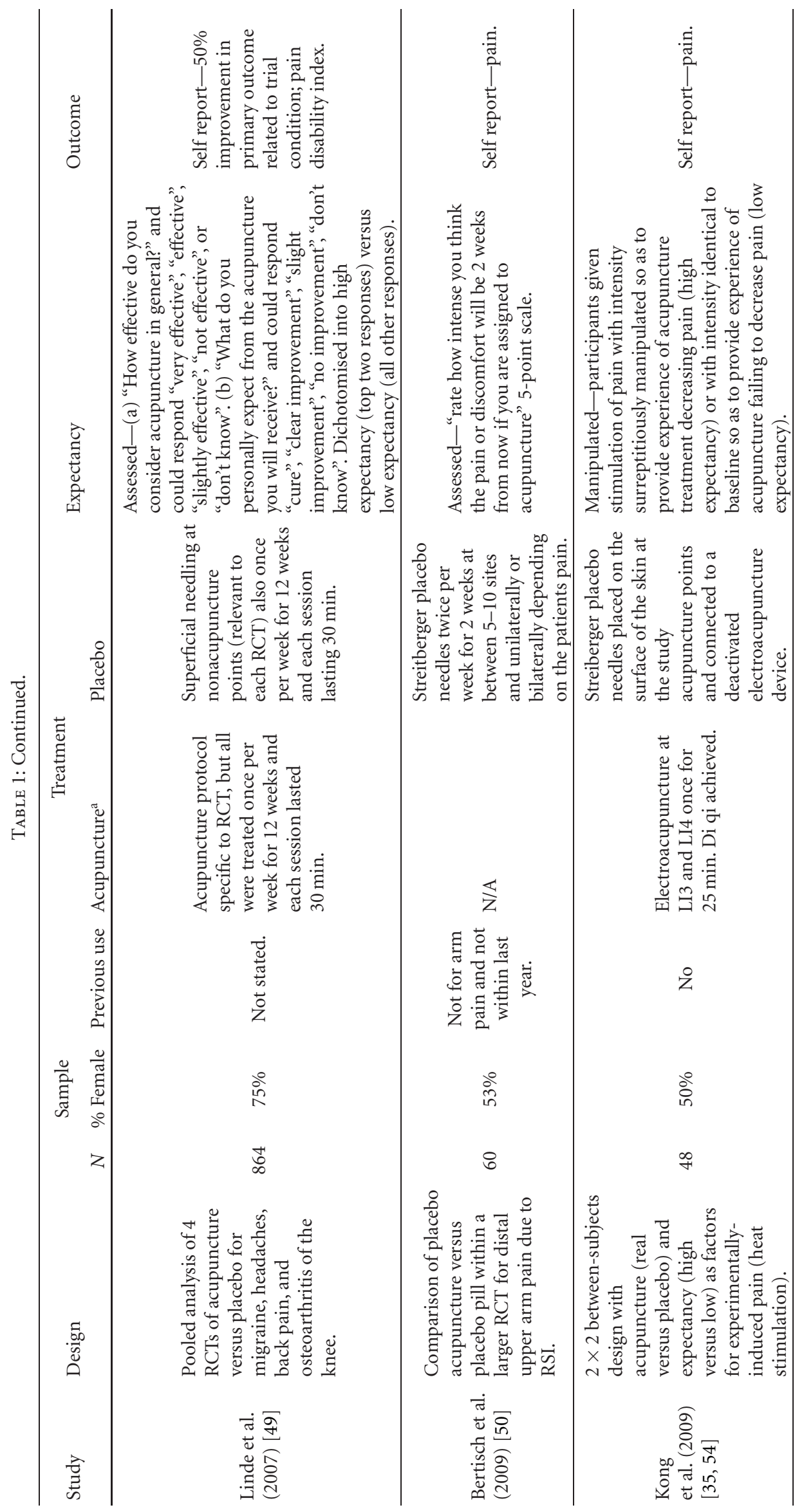




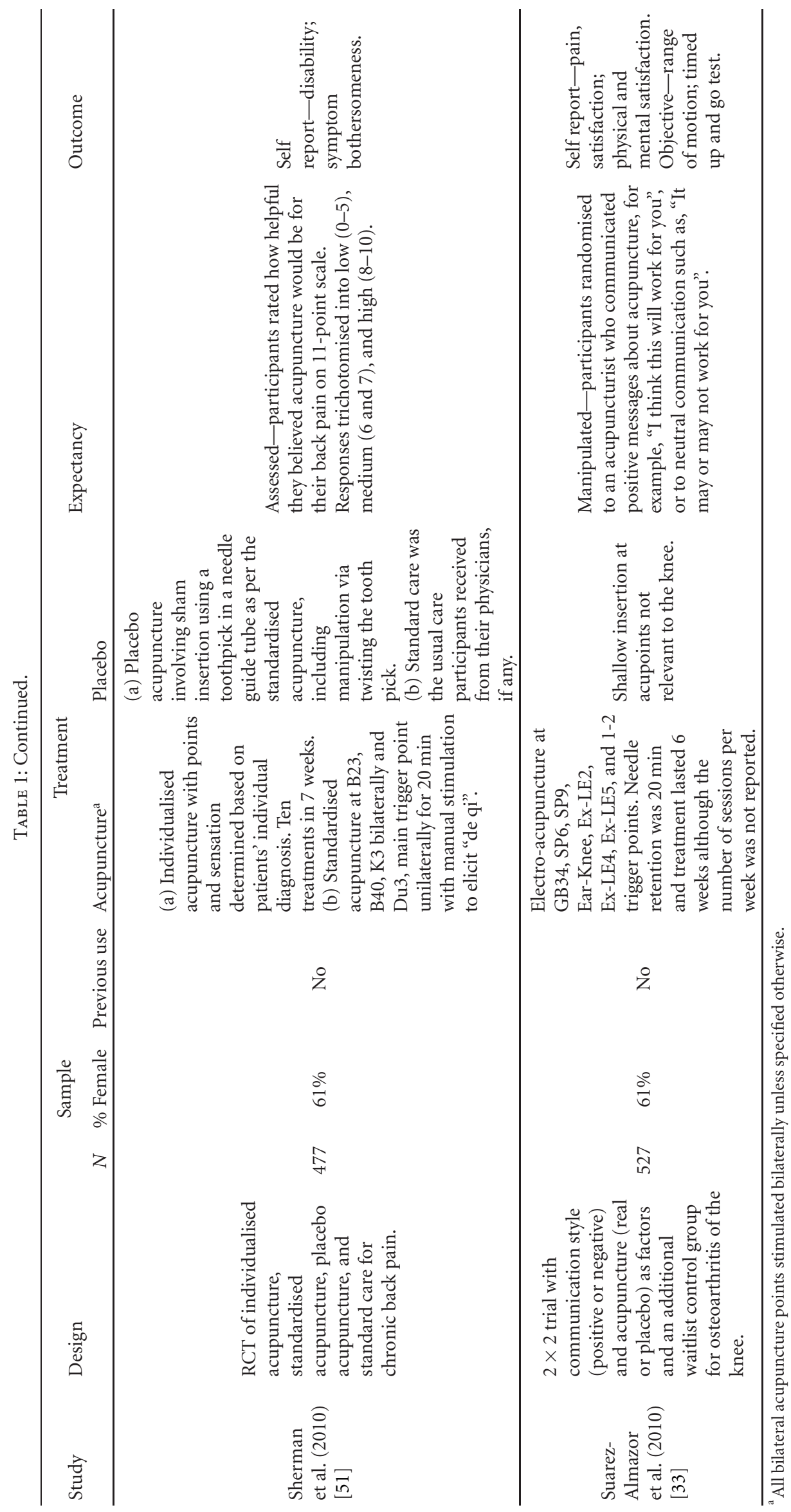


TABLE 2: Summary of included studies' results.

\begin{tabular}{|c|c|c|}
\hline Study & Expectancy & Summary of results ${ }^{\mathrm{a}}$ \\
\hline Berk et al. [48] & Manipulated & $\begin{array}{l}\text { There were no significant differences between real and placebo } \\
\text { acupuncture. There were also no significant differences on shoulder } \\
\text { mobility for those given positive versus negative information about } \\
\text { acupuncture. Those given positive information reported lower shoulder } \\
\text { pain than those given negative information, but this did not reach } \\
\text { statistical significance }(P=0.053) \text {. Interaction between acupuncture and } \\
\text { expectancy not reported. }\end{array}$ \\
\hline
\end{tabular}

There were no significant main effects of acupuncture or expectancy. However, posttreatment experimentally-induced pain reduced significantly Knox et al. (1979) [52] Manipulated from baseline in participants given real acupuncture with positive information but not in participants given real acupuncture with variable or negative information, nor in participants given placebo acupuncture with positive, variable, or negative information.

There was a significant interaction between acupuncture and expectancy. Simple effects revealed participants receiving real acupuncture reported significantly less experimentally-induced pain if they had "high

Norton et al. (1984) [53] Assessed (dichotomised) expectancy" compared with "low expectancy". Participants with "high expectancy" who received real acupuncture also reported significantly less pain than those also with "high expectancy" but who received placebo acupuncture. Main effects of acupuncture and expectancy not reported.

There were no significant differences on any angina outcome between

Ballegaard et al. (1995) [55] Assessed (dichotomised) participants categorised as having "maximal expectancy" and "submaximal expectancy". Main effect of acupuncture and its interaction with expectancy not reported.

Those receiving real acupuncture were more likely to respond to treatment than those receiving placebo acupuncture. Higher expectancies for acupuncture's efficacy in general and specifically for the patients' presenting condition were associated with a higher likelihood of

Linde et al. (2007) [49] Assessed (dichotomised) experiencing a 50\% improvement in the studies' main outcome and a reduction in pain disability index both immediately posttreatment and at follow up. Significant interaction on "some" outcomes indicating the improved outcomes for those with "high expectancy" compared with "low expectancy" were more marked for patients receiving real acupuncture than those receiving placebo acupuncture.

No significant relationship was found between expectancies and upper arm

Bertisch et al. (2009) [50] Assessed pain following placebo acupuncture in both unadjusted and multivariate analysis.

No main effect of acupuncture. Participants allocated to receive pre-conditioning consistent with acupuncture having an analgesic effect Kong et al. (2009) [35, 54] Manipulated reported significantly less experimentally-induced pain following acupuncture than those allocated to receive pre-conditioning of acupuncture having no effect. There was no interaction between acupuncture and expectancy.

Individualised, standardised, and placebo acupuncture were more effective at reducing chronic low back pain than usual care, but there were no

Sherman et al. (2010) [51] Assessed (trichotomised) significant differences among these three treatments. There were also no significant differences between those with "high", "medium", and "low" expectancies. Interaction between treatment and expectancy not reported.

No differences were found between real and placebo acupuncture, but both led to better outcomes compared with the waitlist control group.

Suarez-Almazor et al. (2010) [33]

Manipulated
Participants allocated to receive positive information had significantly lower pain and higher satisfaction than those allocated to receive neutral information and this was independent of whether real or placebo acupuncture was administered.

\footnotetext{
${ }^{a}$ All results are main effects unless stated otherwise.
} 
TABLE 3: Risk of bias assessment for the included studies.

\begin{tabular}{|c|c|c|c|c|c|c|c|}
\hline \multirow{2}{*}{ Study } & \multirow{2}{*}{$\begin{array}{l}\text { Adequate } \\
\text { sequence } \\
\text { generation? }\end{array}$} & \multirow{2}{*}{$\begin{array}{c}\text { Allocation } \\
\text { Concealment? }\end{array}$} & \multicolumn{2}{|c|}{ Blinding? } & \multirow{2}{*}{$\begin{array}{l}\text { Incomplete } \\
\text { data } \\
\text { addressed? }\end{array}$} & \multirow{2}{*}{$\begin{array}{r}\text { Free of selective } \\
\text { reporting bias? }\end{array}$} & \multirow{2}{*}{ Free of other bias? } \\
\hline & & & Participant & $\begin{array}{l}\text { Outcome } \\
\text { Assessor }\end{array}$ & & & \\
\hline $\begin{array}{l}\text { Berk et al. } \\
\text { (1977) [48] }\end{array}$ & Unclear & Unclear & Yes & Unclear & Yes & Yes & Yes \\
\hline $\begin{array}{l}\text { Knox et al. } \\
\text { (1979) [52] }\end{array}$ & No & No & Yes & Yes & Yes & Yes & Yes \\
\hline $\begin{array}{l}\text { Norton et al. } \\
(1984)[53]\end{array}$ & Unclear & Yes & Yes & Unclear & Yes & Yes & $\begin{array}{l}\text { No-small sample size for } \\
\text { correlational study; dichotomised } \\
\text { expectancy }\end{array}$ \\
\hline $\begin{array}{l}\text { Ballegaard et al. } \\
\text { (1995) [55] }\end{array}$ & Unclear & Unclear & Yes & Yes & Yes & Unclear & $\begin{array}{l}\text { No-small sample size for } \\
\text { correlational study; dichotomised } \\
\text { expectancy }\end{array}$ \\
\hline $\begin{array}{l}\text { Linde et al. } \\
\text { (2007) [49] }\end{array}$ & Yes & Yes & Yes & Unclear & Yes & Yes & No-dichotomised expectancy \\
\hline $\begin{array}{l}\text { Bertisch et al. } \\
(2009)[50]\end{array}$ & Yes & Yes & Yes & Yes & Yes & Yes & $\begin{array}{l}\text { No-small-medium sample size } \\
\text { for correlational study }\end{array}$ \\
\hline $\begin{array}{l}\text { Kong et al. } \\
(2009)[35,54]\end{array}$ & Unclear & Unclear & Yes & Unclear & Yes & Yes & Yes \\
\hline $\begin{array}{l}\text { Sherman et al. } \\
(2010)[51]\end{array}$ & Yes & Yes & Yes & Yes & Yes & Yes & No-trichotomised expectancy \\
\hline $\begin{array}{l}\text { Suarez-Almazor } \\
\text { et al. (2010) [33] }\end{array}$ & Yes & Yes & Yes & Yes & Yes & Yes & Yes \\
\hline
\end{tabular}

${ }^{a}$ Risk of bias for blinding was assessed only for whether participants were intended to be blind to the type of acupuncture they received (real or placebo) and whether outcome assessors were blind to the participants' allocation. Blinding of acupuncturists regarding acupuncture treatment is not possible, nor is it possible to blind participants regarding an expectancy manipulation; therefore, these were not included in the risk of bias assessment. ${ }^{\mathrm{b}}$ In Bertisch et al. [50], even though only placebo acupuncture was delivered for the period of interest, they were told they may receive real or placebo acupuncture and are, therefore, considered as blind to treatment allocation.

simplified their expectancy assessment via dichotomisation or trichotomisation and three studies $[49,51,53,55]$ had relatively small sample sizes given their correlational nature $[50,53,55]$.

\section{Discussion}

Given that patient expectancies are often proposed to be a key factor in acupuncture's effectiveness compared with no treatment or standard care $[28,29]$, relatively few studies have examined the relationship between expectancies and treatment responses following acupuncture. Our systematic search identified only 14 unique studies testing the relationship between patient expectancies and outcomes following acupuncture needling, of which nine met our criteria for inclusion. The high level of heterogeneity across studies and incomplete reporting in some meant that meta-analysis was not possible. A descriptive review revealed that while there was evidence of a significant relationship between patient expectancies and acupuncture needling outcomes in some studies, others failed to find these effects. The pattern of results suggested that studies on experimentallyinduced pain, that manipulated expectancies, or those involving electroacupuncture were more likely to find a significant relationship. However, caution is required in generalising these results, as it was more common for studies on experimentally-induced pain to manipulate expectancies and to employ electro-acupuncture, meaning that the effects of each cannot be disentangled on the basis of the available data. Further, the largest study on a clinical outcome, that assessed expectancies, and that involved manual acupuncture, did find evidence of a significant relationship between expectancy and acupuncture outcomes [49]. It was also the case that some studies were at higher risk of bias than others.

The differences in study design and inconsistent results across the identified studies raise important considerations regarding which methodological approach is best equipped to determine the contribution of patient expectancies to acupuncture outcomes. The two most pertinent methodological issues are (1) whether to assess or manipulate expectancies and (2) how to accurately assess expectancies.

Of the nine studies identified here, five assessed expectancies $[49-51,53,55]$ and four manipulated expectancies [33, $48,52,54]$. Studies that involve manipulating expectancies are better able to determine how patient expectancies contribute to acupuncture outcomes because of their experimental nature and might be considered superior for this reason. However, studies that only manipulate expectancies are entirely reliant on the ability of the manipulation to influence expectancies. This leads to problems determining whether an unsuccessful manipulation failed because it did not sufficiently influence expectancies or because the participants' 
expectancies had no effect on their treatment response, as is the case in Berk et al.'s [48] study. Studies that assess expectancies have the advantage of being able to directly evaluate the relationship between expectancy and acupuncture outcomes, thereby overcoming problems to do with relying on the efficacy of an expectancy manipulation. However, these types of studies might be considered a weaker source of evidence because they are correlational in nature.

An apparently simple way to overcome this issue is to include an assessment of expectancy in studies involving manipulations. However, there are a number of other potential limitations associated with assessing expectancies that need consideration. First, questioning participants about their expectancies regarding acupuncture's efficacy could undermine the study's validity if it influences what they expect or if it makes them question the purpose of the study. Second, determining the best time to assess expectancies is also difficult. Assessing them immediately before the first acupuncture treatment provides a prospective assessment, but expectancies may change during the course of the treatment, especially if it lasts for more than a few days. On the other hand, assessing expectancies immediately before or immediately after the outcomes are assessed could lead to priming that artificially inflates the strength of the relationship between expectancy and the outcome. Thirdly, there have been few systematic attempts to develop methods of assessing expectancies, both within acupuncture research and in the placebo literature more broadly. Most of the studies that assessed expectancies identified here used a single expectancy item. For the most part, these were 5-point Likert-type scales, although, as can be seen in Table 1, both the wording of the question and the labels for the response options varied considerably. It was also common for studies assessing expectancies to dichotomise [49, 53, 55], or in one case trichotomise [51], patients' responses into different levels of expectancy, however, categorising such variables has been heavily criticised, because it can substantially reduce statistical power [57-59].

Therefore, while studies that both manipulate and assess expectancies are best able to test the relationship between expectancy and acupuncture outcomes, questions regarding the influence of asking patients to report their expectancies and both when and how expectancies should be assessed need to be addressed empirically in order to determine the most appropriate method of assessing expectancies. Of course, it may not always be practical to incorporate an expectancy manipulation into a trial of acupuncture, as this may require substantially larger samples to achieve the same level of power or may raise ethical considerations if deception is required. In these circumstances, it is still useful to assess expectancies as this can provide estimates of the relationship between expectancy and treatment responses following acupuncture, but again, the best methods of assessing expectancy need to be tested empirically in order to maximise the validity of such research.

There are three potential limitations to the current review. Firstly, as noted above, we were unable to conduct meta-analysis to estimate and test the effect size for the relationship between expectancy and acupuncture outcomes due to the high heterogeneity in methodology and incomplete reporting in some studies. While this does mean that we were unable to determine an average effect size across studies, the descriptive review provided here does highlight a number of important methodological considerations that will inform future research in this area. Secondly, as with most systematic reviews, there is the possibility of publication bias. In the current case, this could mean that studies failing to find a statistically significant relationship between expectancy and acupuncture outcomes were less likely to be published than those finding statistically significant effects, which may lead to overestimation of the influence of expectancy. We, therefore, encourage researchers conducting RCTs of acupuncture to report, even briefly, of any failures to find a significant relationship between expectancy and acupuncture outcomes. Finally, only papers published in English were reviewed, meaning that other relevant studies may be published in other languages.

In summary, there have been relatively few research studies testing the relationship between expectancy and acupuncture outcomes. While there did appear to be evidence for a significant relationship between patient expectancies and treatment responses following acupuncture, there were some inconsistencies across studies. Future studies attempting to address this question should, where possible, both manipulate and assess expectancies. However, considerations regarding currently used methods of assessing expectancy, such as timing and wording of the questions, need to be addressed first in order to establish the best approach and to ensure the validity of these assessments and any conclusions drawn about the relationship between expectancy and acupuncture outcomes. Further, investigating potential moderators of the relationship between expectancy and acupuncture outcomes, such as type of acupuncture (real versus placebo), type of stimulation (manual versus electroacupuncture) would prove useful for better understanding the circumstances under which expectancies can influence treatment responses following acupuncture.

\section{Acknowledgments}

We have no conflicts of interest in producing this review. No funding was obtained for the review.

\section{References}

[1] D. C. Cherkin, K. J. Sherman, A. L. Avins et al., "A randomized trial comparing acupuncture, simulated acupuncture, and usual care for chronic low back pain," Archives of Internal Medicine, vol. 169, no. 9, pp. 858-866, 2009.

[2] M. Haake, H. H. Müller, C. Schade-Brittinger et al., "German Acupuncture Trials (GERAC) for chronic low back pain: randomized, multicenter, blinded, parallel-group trial with 3 groups," Archives of Internal Medicine, vol. 167, no. 17, pp. 1892-1898, 2007.

[3] N. Sahin, E. Ozcan, K. Sezen, O. Karatas, and H. Issever, "Efficacy of acupunture in patients with chronic neck pain-a randomised, sham controlled trial," Acupuncture \& ElectroTherapeutics Research, vol. 35, no. 1-2, pp. 17-27, 2010. 
[4] A. J. Lembo, L. Conboy, J. M. Kelley et al., "A treatment trial of acupuncture in IBS patients," American Journal of Gastroenterology, vol. 104, no. 6, pp. 1489-1497, 2009.

[5] B. Colagiuri, "Participant expectancies in double-blind randomized placebo-controlled trials: potential limitations to trial validity," Clinical Trials, vol. 7, no. 3, pp. 246-255, 2010.

[6] H. MacPherson, L. Thorpe, and K. Thomas, "Beyond needling - therapeutic processes in acupuncture care: a qualitative study nested within a low-back pain trial," Journal of Alternative and Complementary Medicine, vol. 12, no. 9, pp. 873-880, 2006.

[7] C. Paterson and P. Dieppe, "Characteristic and incidental (placebo) effects in complex interventions such as acupuncture," British Medical Journal, vol. 330, no. 7501, pp. 1202 1205, 2005.

[8] P. White, K. Linde, and R. S. Schnyer, "Investigating the components of acupuncture treatment," in Acupuncture Research: Strategies for Establishing and Evidence Base, H. MacPherson et al., Ed., pp. 133-152, Churchill Livingstone, Philadelphia, Pa, USA, 2008.

[9] C. M. Cassidy, "Moffet on the similarity of response to "active" and "sham" acupuncture," Journal of Alternative and Complementary Medicine, vol. 15, no. 3, pp. 209-210, 2009.

[10] C. Ritenbaugh, M. Verhoef, S. Fleishman, H. Boon, and A. Leis, "Whole systems research: a discipline for studying complementary and alternative medicine," Alternative Therapies in Health and Medicine, vol. 9, no. 4, pp. 32-36, 2003.

[11] H. MacPherson and K. Thomas, "Self-help advice as a process integral to traditional acupuncture care: implications for trial design," Complementary Therapies in Medicine, vol. 16, no. 2, pp. 101-106, 2008.

[12] I. Lund and T. Lundeberg, "Are minimal, superficial or sham acupuncture procedures acceptable as inert placebo controls?" Acupuncture in Medicine, vol. 24, no. 1, pp. 13-15, 2006.

[13] T. Lundeberg, I. Lund, J. Näslund, and M. Thomas, "The Emperor's sham-wrong assumption that sham needling is sham," Acupuncture in Medicine, vol. 26, no. 4, pp. 239-242, 2008.

[14] T. Lundeberg, I. Lund, A. Sing, and J. Näslund, "Is placebo acupuncture what it is intended to be," Evidence-Based Complementary and Alternative Medicine, vol. 2011, Article ID 932407, 5 pages, 2011.

[15] S. Stewart-Williams and J. Podd, "The placebo effect: dissolving the expectancy versus conditioning debate," Psychological Bulletin, vol. 130, no. 2, pp. 324-340, 2004.

[16] F. Benedetti, H. S. Mayberg, T. D. Wager, C. S. Stohler, and J. K. Zubieta, "Neurobiological mechanisms of the placebo effect," Journal of Neuroscience, vol. 25, no. 45, pp. 10390-10402, 2005.

[17] F. Benedetti, A. Pollo, L. Lopiano, M. Lanotte, S. Vighetti, and I. Rainero, "Conscious expectation and unconscious conditioning in analgesic, motor, and hormonal placebo/nocebo responses," Journal of Neuroscience, vol. 23, no. 10, pp. 43154323, 2003.

[18] G. H. Montgomery and I. Kirsch, "Classical conditioning and the placebo effect," Pain, vol. 72, no. 1-2, pp. 107-113, 1997.

[19] A. Pollo, M. Amanzio, A. Arslanian, C. Casadio, G. Maggi, and F. Benedetti, "Response expectancies in placebo analgesia and their clinical relevance," Pain, vol. 93, no. 1, pp. 77-84, 2001.

[20] N. J. Voudouris, C. L. Peck, and G. Coleman, "Conditioned placebo responses," Journal of Personality and Social Psychology, vol. 48, no. 1, pp. 47-53, 1985.

[21] N. J. Voudouris, C. L. Peck, and G. Coleman, "Conditioned response models of placebo phenomena: further support," Pain, vol. 38, no. 1, pp. 109-116, 1989.
[22] N. J. Voudouris, C. L. Peck, and G. Coleman, "The role of conditioning and verbal expectancy in the placebo response," Pain, vol. 43, no. 1, pp. 121-128, 1990.

[23] D. G. Finniss, T. J. Kaptchuk, F. Miller, and F. Benedetti, "Biological, clinical, and ethical advances of placebo effects," The Lancet, vol. 375, no. 9715, pp. 686-695, 2010.

[24] I. Kirsch, Sapirstein G Listening to Prozac but Hearing Placebo: A Meta-Analysis of Antidepressant Medication, Prevention \& Treatment, Washington, DC, USA, 1998.

[25] M. Suetsugi, Y. Mizuki, K. Yamamoto, S. Uchida, and Y. Watanabe, "The effect of placebo administration on the firstnight effect in healthy young volunteers," Progress in NeuroPsychopharmacology and Biological Psychiatry, vol. 31, no. 4, pp. 839-847, 2007.

[26] B. Colagiuri and R. A. Boakes, "Perceived treatment, feedback, and placebo effects in double-blind RCTs: an experimental analysis," Psychopharmacology, vol. 208, no. 3, pp. 433-441, 2010.

[27] F. Benedetti, G. Maggi, L. Lopiano et al., Open Versus Hidden Medical Treatments: The Patient's Knowledge about a Therapy Affects the Therapy Outcome, Prevention \& Treatment, Washington, DC, USA, 2003.

[28] N. E. O'Connell, B. M. Wand, and B. Goldacre, "Interpretive bias in acupuncture research?: a case study," Evaluation and the Health Professions, vol. 32, no. 4, pp. 393-409, 2009.

[29] R. Barker Bausell, L. Lao, S. Bergman, W. L. Lee, and B. M. Berman, "Is acupuncture analgesia an expectancy effect? Preliminary evidence based on participants' perceived assignments in two placebo-controlled trials," Evaluation and the Health Professions, vol. 28, no. 1, pp. 9-26, 2005.

[30] A. J. M. De Craen, D. E. Moerman, S. H. Heisterkamp, G. N. J. Tytgat, J. G. P. Tijssen, and J. Kleijnen, "Placebo effect in the treatment of duodenal ulcer," British Journal of Clinical Pharmacology, vol. 48, no. 6, pp. 853-860, 1999.

[31] A. J. M. De Craen, J. G. P. Tijssen, J. De Gans, and J. Kleijnen, "Placebo effect in the acute treatment of migraine: subcutaneous placebos are better than oral placebos," Journal of Neurology, vol. 247, no. 3, pp. 183-188, 2000.

[32] J. Neyman and E. S. Pearson, "The testing of statistical hypotheses in relation to probabilities a priori," Mathematical Proceedings of the Cambridge Philosophical Society, vol. 29, pp. 492-510, 1933.

[33] M. E. Suarez-Almazor, C. Looney, Y. Liu et al., "A randomized controlled trial of acupuncture for osteoarthritis of the knee: effects of patient-provider communication," Arthritis Care and Research, vol. 62, no. 9, pp. 1229-1236, 2010.

[34] M. E. Moore and S. N. Berk, "Acupuncture for chronic shoulder pain. An experimental study with attention to the role of placebo and hypnotic susceptibility," Annals of Internal Medicine, vol. 84, no. 4, pp. 381-384, 1976.

[35] J. Kong, T. J. Kaptchuk, G. Polich et al., "Expectancy and treatment interactions: a dissociation between acupuncture analgesia and expectancy evoked placebo analgesia," NeuroImage, vol. 45, no. 3, pp. 940-949, 2009.

[36] C. E. Zyloney, K. Jensen, G. Polich et al., "Imaging the functional connectivity of the Periaqueductal Gray during genuine and sham electroacupuncture treatment," Molecular Pain, vol. 6, article 80, 2010.

[37] N. E. Foster, E. Thomas, J. C. Hill, and E. M. Hay, "The relationship between patient and practitioner expectations and preferences and clinical outcomes in a trial of exercise and acupuncture for knee osteoarthritis," European Journal of Pain, vol. 14, no. 4, pp. 402-409, 2010. 
[38] D. W. So, "Acupuncture outcomes, expectations, patientprovider relationship, and the placebo effect: implications for health promotion," American Journal of Public Health, vol. 92, no. 10, pp. 1662-1667, 2002.

[39] A. D. Wasan, J. Kong, L. D. Pham, T. J. Kaptchuk, R. Edwards, and R. L. Gollub, "The impact of placebo, psychopathology, and expectations on the response to acupuncture needling in patients with chronic low back pain," Journal of Pain, vol. 11, no. 6, pp. 555-563, 2010.

[40] M. Karst, D. Schneidewind, D. Scheinichen et al., "Acupuncture induces a pro-inflammatory immune response intensified by a conditioning-expectation effect," Forschende Komplementarmedizin, vol. 17, no. 1, pp. 21-27, 2010.

[41] A. Liberati, D. G. Altman, J. Tetzlaff et al., "The PRISMA statement for reporting systematic reviews and meta-analyses of studies that evaluate health care interventions: explanation and elaboration," PLoS Medicine, vol. 6, no. 7, Article ID e1000100, 2009.

[42] D. Moher, A. Liberati, J. Tetzlaff et al., "Preferred reporting items for systematic reviews and meta-analyses: the PRISMA statement," PLoS Medicine, vol. 6, no. 7, Article ID e1000097, 2009.

[43] S. Greenland, "Invited commentary: a critical look at some popular meta-analytic methods," American Journal of Epidemiology, vol. 140, no. 3, pp. 290-296, 1994.

[44] S. Greenland, "Quality scores are useless and potentially misleading: reply to 're: a critical look at some popular analytic methods", American Journal of Epidemiology, vol. 140, no. 3, pp. 300-301, 1994.

[45] J. D. Emerson, E. Burdick, D. C. Hoaglin, F. Mosteller, and T. C. Chalmers, "An empirical study of the possible relation of treatment differences to quality scores in controlled randomized clinical trials," Controlled Clinical Trials, vol. 11, no. 5, pp. 339-352, 1990.

[46] P. Jüni, A. Witschi, R. Bloch, and M. Egger, "The hazards of scoring the quality of clinical trials for meta-analysis," Journal of the American Medical Association, vol. 282, no. 11, pp. 10541060, 1999.

[47] J. P. Higgins and S. G. Green, Eds., Cochrane Handbook for Systematic Reviews of Interventions, John Wiley \& Sons, West Sussex, UK, 2008.

[48] S. N. Berk, M. E. Moore, and J. H. Resnick, "Psychosocial factors as mediators of acupuncture therapy," Journal of Consulting and Clinical Psychology, vol. 45, no. 4, pp. 612-619, 1977.

[49] K. Linde, C. M. Witt, A. Streng et al., "The impact of patient expectations on outcomes in four randomized controlled trials of acupuncture in patients with chronic pain," Pain, vol. 128, no. 3, pp. 264-271, 2007.

[50] S. M. Bertisch, A. R. T. Legedza, R. S. Phillips et al., "The impact of psychological factors on placebo responses in a randomized controlled trial comparing sham device to dummy pill," Journal of Evaluation in Clinical Practice, vol. 15, no. 1, pp. 14-19, 2009.

[51] K. J. Sherman, D. C. Cherkin, L. Ichikawa et al., "Treatment expectations and preferences as predictors of outcome of acupuncture for chronic back pain," Spine, vol. 35, no. 15, pp. 1471-1477, 2010.

[52] V. J. Knox, C. E. Handfield-Jones, and K. Shum, "Subject expectancy and the reduction of cold pressor pain with acupuncture and placebo acupuncture," Psychosomatic Medicine, vol. 41, no. 6, pp. 477-486, 1979.
[53] G. Norton, L. Goszer, H. Strub, and S. Man, "The effects of belief on acupuncture analgesia," Canadian Journal of Behavioural Science, vol. 16, pp. 22-29, 1984.

[54] J. Kong, T. J. Kaptchuk, G. Polich et al., "An fMRI study on the interaction and dissociation between expectation of pain relief and acupuncture treatment," NeuroImage, vol. 47, no. 3, pp. 1066-1076, 2009.

[55] S. Ballegaard, B. Karpatschoff, J. A. Holck, C. N. Meyer, and W. Trojaborg, "Acupuncture in angina pectoris: do psychosocial and neurophysiological factors relate to the effect?" Acupuncture \& Electro-Therapeutics Research, vol. 20, no. 2, pp. 101-116, 1995.

[56] M. Borenstein, L. V. Hedges, J. P. Higgins, and H. Rothstein, Introduction to Meta-Analysis, John Wiley \& Sons, West Sussex, UK, 2009.

[57] J. Cohen, "The cost of dichotomization," Applied Psychological Measurement, vol. 7, pp. 249-253, 1986.

[58] D. G. Altman and P. Royston, "The cost of dichotomising continuous variables," British Medical Journal, vol. 332, no. 7549, article 1080, 2006.

[59] P. Royston, D. G. Altman, and W. Sauerbrei, "Dichotomizing continuous predictors in multiple regression: a bad idea," Statistics in Medicine, vol. 25, no. 1, pp. 127-141, 2006. 


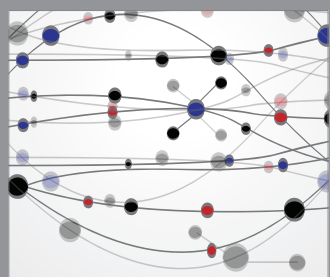

The Scientific World Journal
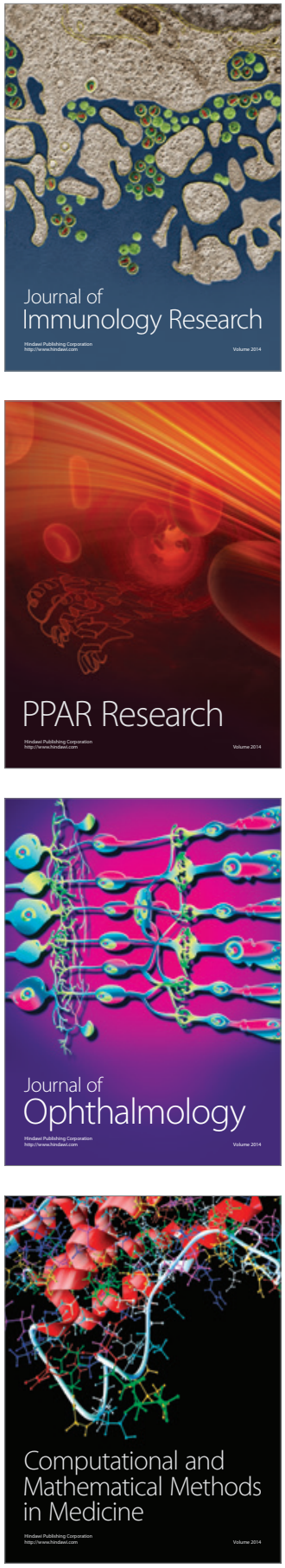

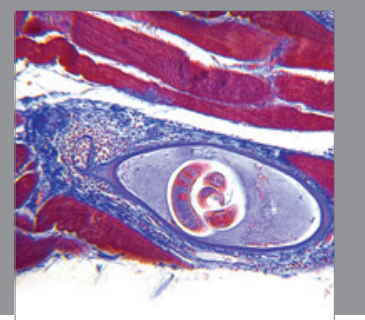

Gastroenterology

Research and Practice
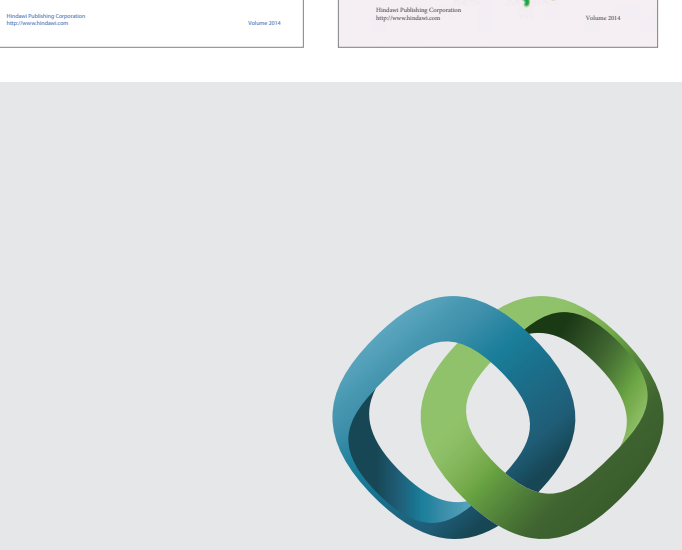

\section{Hindawi}

Submit your manuscripts at

http://www.hindawi.com
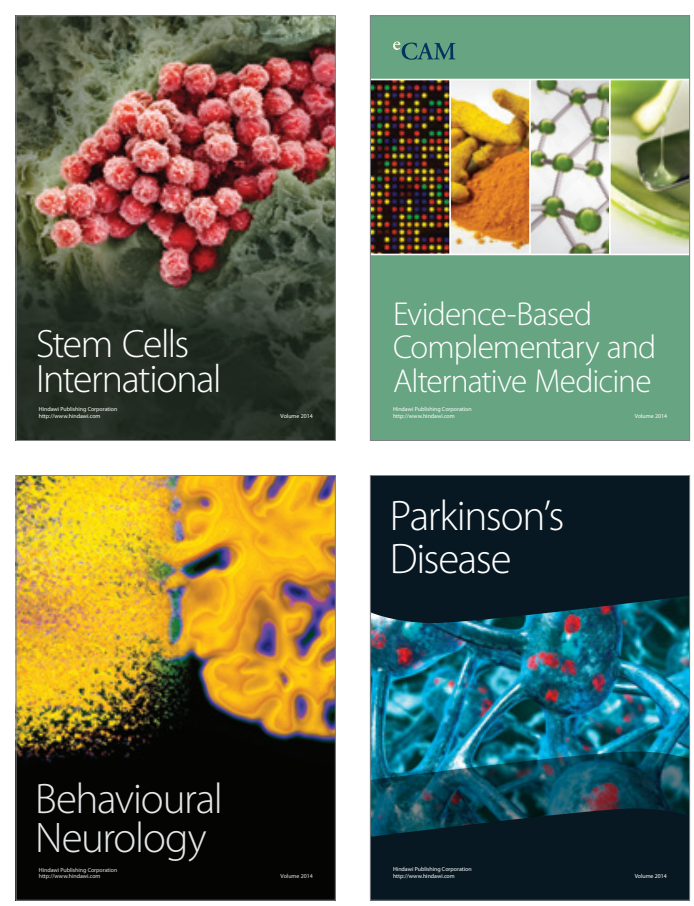

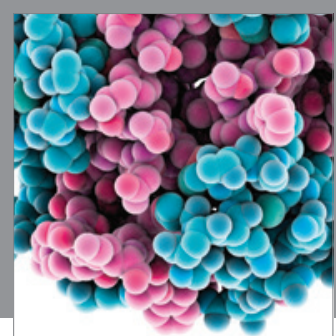

Journal of
Diabetes Research

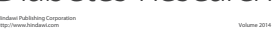

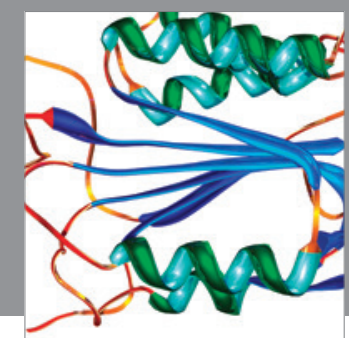

Disease Markers
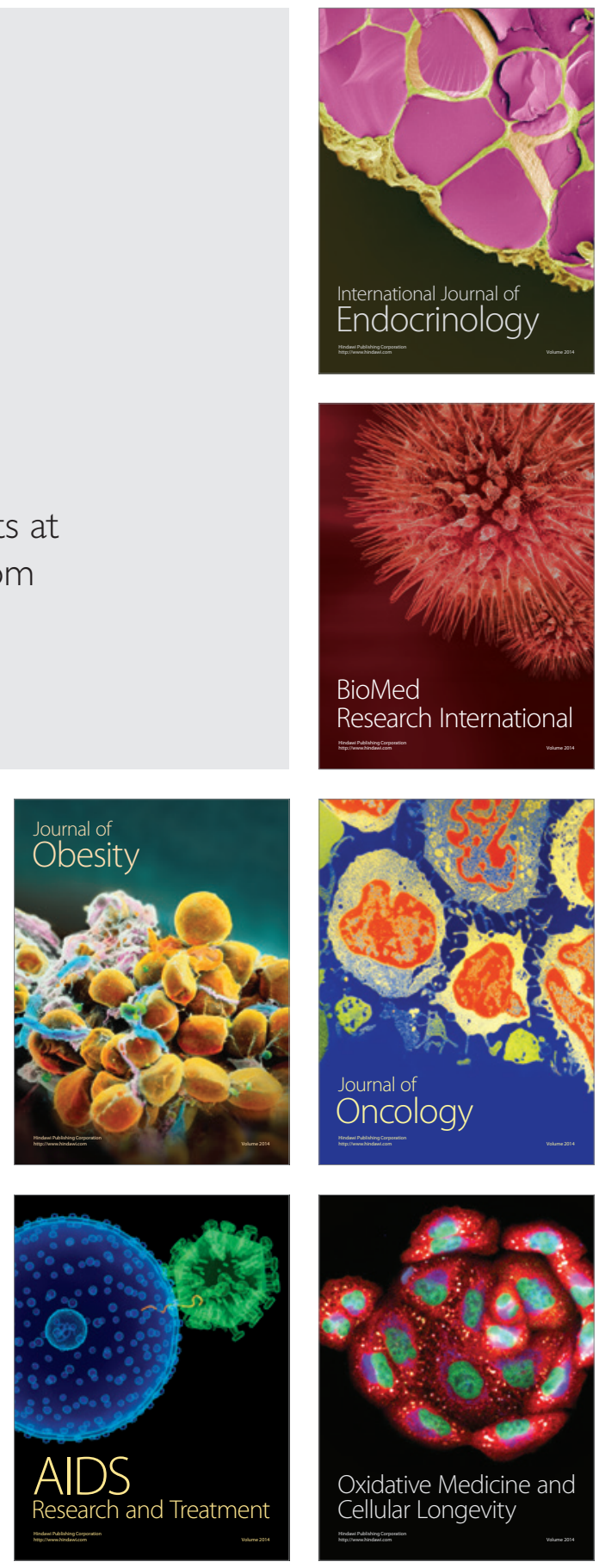